\title{
Isogeometric analysis in electromagnetics: B-splines approximation
}

\author{
A. Buffa*, G. Sangalli ${ }^{\dagger *}$ R. Vázquez* \\ June 6, 2009
}

\begin{abstract}
We introduce a new discretization scheme for Maxwell equations in two space dimension. Inspired by the new paradigm of Isogeometric analysis introduced in [16], we propose an algorithm based on the use of bivariate B-splines spaces suitably adapted to electromagnetics. We construct B-splines spaces of variable interelement regularity on the parametric domain. These spaces (and their push-forwards on the physical domain) form a De Rham diagram and we use them to solve the Maxwell source and eigen problem. Our scheme has the following features: $(i)$ is adapted to treat complex geometries, (ii) is spectral correct, (iii) provides regular (e.g., globally $C^{0}$ ) discrete solutions of Maxwell equations.
\end{abstract}

\section{Introduction}

Isogeometric Analysis was introduced in [16] and aim at improving the connection between numerical simulation and Computer Aided Design (CAD). Then, its potential has been shown in the recent engineering literature (e.g., $[3,5,14]$ ). The main idea of the IGA methodology is to use directly the geometry provided by CAD in terms of non-uniform rational B-splines (NURBS) (see e.g., [20]) and to approximate the unknown solutions of differential operators by the same type of functions. Isogeometric Analysis offers several advantages when compared to the finite elements method. First of all, complicated geometries are represented more accurately, and some common geometries as circles or ellipses are described exactly. Moreover, the description of the geometry is given at the coarsest mesh level, and comes directly from the CAD system. In fact, this eliminates the necessity of further communication with the CAD, when mesh refinement is carried out. This is carried out without changing the geometry. Another advantage is that, apart from the standard $h$ - and $p$-refinements, in [16] the authors introduced the possibility of $k$-refinement, which yields good convergence rates with less degrees of freedom. Finally, Isogeometric Analysis provides smoother functions than finite element methods. This grants improved accuracy (see [11,13]), and has been proved to be particularly interesting in the approximation of the entire spectrum of the Laplace and Bilaplace operators (in [17]).

Our aim is to adapt Isogeometric Analysis to the numerical solution of Maxwell's equations, and in particular to the approximation of the space $\mathbf{H}(\operatorname{curl} ; \Omega)$. In this work, $\Omega$ will be

\footnotetext{
* Istituto di Matematica Applicata e Tecnologie Informatiche del CNR; Via Ferrata, 1; 27100 Pavia; Italy. E-mail: annalisa@imati.cnr.it, vazquez@imati.cnr.it

${ }^{\dagger}$ Dipartimento di Matematica,Università di Pavia; Via Ferrata, 1; 27100 Pavia; Italy. Email:giancarlo.sangalli@unipv . it
} 
a two dimensional, bounded, Lispschitz, simply connected domain and the function spaces are defined in Section 2.

We will focus on the following eigenvalue problem:

Find $\omega \in \mathbb{R}$ and $\mathbf{u} \in \mathbf{H}_{0}(\operatorname{curl} ; \Omega), \mathbf{u} \neq \mathbf{0}$, satisfying

$$
(\operatorname{curl} \mathbf{u}, \operatorname{curl} \mathbf{v})=\omega^{2}(\mathbf{u}, \mathbf{v}) \quad \forall \mathbf{v} \in \mathbf{H}_{0}(\operatorname{curl} ; \Omega) \text {. }
$$

An equivalent formulation of (1) is obtained by imposing a divergence free condition in the space, i.e., seeking the solution in the space $\mathbf{H}_{0}(\operatorname{curl} ; \Omega) \cap \mathbf{H}\left(\operatorname{div}^{0} ; \Omega\right)$.

Since [6] it is known that nodal finite elements fail in approximating the space $\mathbf{H}_{0}(\mathrm{curl} ; \Omega)$. The theoretical understanding goes back to Costabel [8], who proved that the space $\mathbf{H}^{1}(\Omega) \cap$ $\mathbf{H}_{0}(\operatorname{curl} ; \Omega)$ is closed in $\mathbf{H}_{0}(\operatorname{curl} ; \Omega) \cap \mathbf{H}(\operatorname{div} ; \Omega)$ when $\Omega$ is a non-convex polygon or polyhedron. On numerical schemes this result means the following: if the variational formulation in use involves the topology of the space $\mathbf{H}_{0}(\operatorname{curl} ; \Omega) \cap \mathbf{H}(\operatorname{div} ; \Omega)$ then no continuous finite elements (indeed, belonging to $\mathbf{H}^{1}(\Omega) \cap \mathbf{H}_{0}(\operatorname{curl} ; \Omega)$ ) can be used. In fact, there are solutions of Maxwell's equations for which no continuous approximant exists in $\mathbf{H}_{0}(\operatorname{curl} ; \Omega) \cap \mathbf{H}(\operatorname{div} ; \Omega)$, for instance, the singular fields in the L-shaped domain (see [10], or Section 5.3 of the present paper).

Numerical schemes based on standard finite elements naturally need some stabilization on the divergence part, due to the lack of coercivity of the bilinear form $\int_{\Omega} \operatorname{curl}(\cdot) \operatorname{curl}(\cdot)$. If this stabilization involves the $L^{2}$-norm of the divergence, the numerical scheme is then not converging for non-convex domains. A remedy to this was proposed by Costabel and Dauge [9] where the control is on a suitable weighted $L^{2}$-norm of the divergence. The scheme proposed in that paper has two branches of eigenvalues: one is the Maxwell one, and the other one is related to the divergence, and is discarded with suitable post-processing. An alternative procedure is to suitably enrich the space of Lagrangian finite elements with singular solutions. For a detailed description of this method we refer to $[2,15]$ and references therein.

Instead, the solution of problem (1) with edge finite elements, introduced by Nédélec [19], is known to be convergent and free of spurious modes [6, 7]. The main feature of edge elements is to solve (1) without requiring any control on the divergence. This is made possible by the validity of the De Rham commuting diagram [1, 18]. As it is well known, edge elements provide, in general, discontinuous approximations of electromagnetic fields, and no control on the divergence of the discrete solutions can be set.

In this work we introduce a new numerical method for the solution of problem (1) in the spirit of [16]. We restrict, for the sake of simplicity, to classical B-splines approximations of the geometry and of the unknown field. To some extend, this method can be understood as a generalization of edge elements to B-splines-based Isogeometric Analysis. In fact, the key point of the method is the choice of adequate spaces of B-splines such that they satisfy the De Rham diagram. With this choice, it is possible to approximate the space $\mathbf{H}_{0}(\operatorname{curl} ; \Omega)$ without any control on the divergence, analogously to what edge elements do. The numerical analysis of this method is not well developed yet, due to the lack of a commuting projector, and we prove here only suboptimal convergence results for regular solutions. However, we have performed several numerical tests which show the optimal behavior of the method. Indeed, in all cases the solution is free of spurious modes, and the method has proved capable to approximate singular solutions with good convergence rate. Moreover, the numerical results also show that for regular solutions the divergence converges to zero as the mesh is refined.

The outline of the paper is as follows. In Section 2 we introduce some notation and recall the continuous problems we want to solve, namely a spectral problem and a given 
source problem derived from Maxwell's equations. In Section 3 we present a brief review on B-splines basis functions and introduce the new discretization scheme, based on B-spline spaces. The approximation analysis of this scheme is carried out in Section 4 following the results of [4]. Finally, in Section 5 we present several numerical tests which confirm the good performance of our method.

\section{The continuous problem}

Let $D \subset \mathbb{R}^{2}$ be a bounded Lipschitz domain. We denote by $L^{p}(D)$ the classical Lebesgue spaces endowed with the norm $\|\cdot\|_{L^{p}(D)}$, and by $\mathbf{L}^{p}(D)$ their vectorial counterparts. The Hilbert spaces $H^{k}(D)$ denote the functions in $L^{2}(D)$ such that their $k$-th order derivatives also belong to $L^{2}(D)$, and by $\mathbf{H}^{k}(D)$ we denote their vectorial counterparts. For $k=0$, $\mathbf{H}^{0}(D)=\mathbf{L}^{2}(D)$. The space $\mathbf{H}($ curl; $D)$ is the space of fields belonging to $\mathbf{L}^{2}(D)$ such that their curl belongs to $L^{2}(D)$, and it is endowed with the norm $\|\mathbf{v}\|_{\mathbf{H}(\operatorname{curl} ; D)}:=\left(\|\mathbf{v}\|_{\mathbf{L}^{2}}^{2}+\right.$ $\|$ curl $\left.\mathbf{v} \|_{L^{2}}^{2}\right)^{1 / 2}$. Analogously, the space $\mathbf{H}($ div; $D)$ is the space of functions in $\mathbf{L}^{2}(D)$ such that their divergence belongs to $L^{2}(D)$, and it is endowed with the norm $\|\mathbf{v}\|_{\mathbf{H}(\operatorname{div} ; D)}:=$ $\left(\|\mathbf{v}\|_{\mathbf{L}^{2}}^{2}+\|\operatorname{div} \mathbf{v}\|_{L^{2}}^{2}\right)^{1 / 2}$. Its subspace $\mathbf{H}\left(\operatorname{div}^{0} ; D\right)$ is formed by all functions in $\mathbf{H}(\operatorname{div} ; D)$ with divergence equal to zero.

Let $\Omega$ be our computational domain, which for the sake of simplicity we assume to be simply connected. We assume that the boundary of $\Omega$ is split into two disjoint parts, $\partial \Omega=\Gamma_{D} \cup \Gamma_{N}$, with $\Gamma_{D} \neq \emptyset$. We denote by $\mathbf{H}_{0, \Gamma_{D}}(\operatorname{curl} ; \Omega)$ the space of functions in $\mathbf{H}(\operatorname{curl} ; \Omega)$ with vanishing tangential trace in $\Gamma_{D}$. In particular, we denote $\mathbf{H}_{0}(\operatorname{curl} ; \Omega):=$ $\mathbf{H}_{0, \partial \Omega}(\operatorname{curl} ; \Omega)$

We are interested in solving two different kind of problems, both of them arising from Maxwell's equations with constant physical properties. The first one is the eigenvalue problem (see, for instance, [6]):

Find $\omega \in \mathbb{R}$ and $\mathbf{u} \in \mathbf{H}_{0}(\operatorname{curl} ; \Omega), \mathbf{u} \neq \mathbf{0}$, satisfying

$$
(\operatorname{curl} \mathbf{u}, \operatorname{curl} \mathbf{v})=\omega^{2}(\mathbf{u}, \mathbf{v}) \quad \forall \mathbf{v} \in \mathbf{H}_{0}(\operatorname{curl} ; \Omega) .
$$

It is well known that $\omega=0$ is the essential spectrum, with associated infinite dimension eigenspace, and that all other eigenvalues form a sequence diverging to infinity with associated eigenspaces belonging to $\mathbf{H}_{0}(\operatorname{curl} ; \Omega) \cap \mathbf{H}\left(\operatorname{div}^{0} ; \Omega\right)$.

The second problem we deal with is deduced from Maxwell's equations with a given current density f, and imposing mixed homogeneous boundary conditions. The equations of the problem in its strong form are the following:

Given $\mathbf{f} \in \mathbf{L}^{2}(\Omega)$, find $\mathbf{u}$ satisfying

$$
\left\{\begin{array}{cl}
\operatorname{curl} \operatorname{curl} \mathbf{u}+\mathbf{u}=\mathbf{f} & \text { in } \Omega \\
\mathbf{u} \times \mathbf{n}=\mathbf{0} & \text { on } \Gamma_{D} \\
\operatorname{curl} \mathbf{u}=0 & \text { on } \Gamma_{N}
\end{array}\right.
$$

And in weak form the problem reads:

Given $\mathbf{f} \in \mathbf{L}^{2}(\Omega)$, find $\mathbf{u} \in \mathbf{H}_{0, \Gamma_{D}}(\operatorname{curl} ; \Omega)$ such that

$$
\int_{\Omega} \operatorname{curl} \mathbf{u} \operatorname{curl} \mathbf{v}+\int_{\Omega} \mathbf{u} \cdot \mathbf{v}=\int_{\Omega} \mathbf{f} \cdot \mathbf{v} \quad \forall \mathbf{v} \in \mathbf{H}_{0, \Gamma_{D}}(\operatorname{curl} ; \Omega) .
$$

The analysis of this problem is already well known, and existence and uniqueness of solution can be proved (see, for instance, [18, Ch. 4]). 


\section{Discretization of the problem using B-splines}

In this section we introduce a discretization scheme based on B-splines for problems (2) and (4), and which satisfies the De Rham diagram. In order to do so, we first present an introduction of B-spline spaces and their basis functions. The aim of this presentation is to establish the notation and recall some properties of B-splines. For a more complete review on this subject we refer the reader to [16]. Further analysis of B-splines functions and spaces can be found in [12] and [21].

\subsection{An overview of B-splines}

Given two positive integers $p$ and $n$, we introduce the (ordered) knot vector

$$
\Xi:=\left\{0=\xi_{1}, \xi_{2}, \ldots, \xi_{n+p+1}=1\right\},
$$

where we allow repetition of knots, that is, we only assume $\xi_{1} \leq \xi_{2} \leq \cdots \leq \xi_{n+p+1}$. We also introduce the vector $\left\{\zeta_{1}, \ldots, \zeta_{m}\right\}$ of knots without repetitions, and the vector $\left\{r_{1}, \ldots, r_{m}\right\}$ of their corresponding multiplicities, such that

$$
\Xi=\{\underbrace{\zeta_{1}, \ldots, \zeta_{1}}_{r_{1} \text { times }}, \underbrace{\zeta_{2}, \ldots, \zeta_{2}}_{r_{2} \text { times }}, \ldots, \underbrace{\zeta_{m}, \ldots, \zeta_{m}}_{r_{m} \text { times }}\}
$$

with $\sum_{i=1}^{m} r_{i}=n+p+1$. The maximum multiplicity we allow is $p+1$. In the following we will only work with open knot vectors, which means that $r_{1}=r_{m}=p+1$, that is, the first $p+1$ knots in $\Xi$ are equal to 0 , and the last $p+1$ are equal to 1 . Notice that this implies $n \geq p+1$.

Through the iterative procedure detailed in [16] we construct $p$-degree (that is, $(p+1)$ order) B-spline basis functions, denoted by $B_{i}$, for $i=1, \ldots, n$. These basis functions are piecewise polynomials of degree $p$ on the subdivision $\left\{\zeta_{1}, \ldots, \zeta_{m}\right\}$. At $\zeta_{i}$ they have $\alpha_{i}:=p-r_{i}$ continuous derivative. Therefore, $-1 \leq \alpha_{i} \leq p-1$ : the maximum multiplicity allowed, $r_{i}=p+1$, gives $\alpha_{i}=-1$, which stands for a discontinuity at $\zeta_{i}$. The vector $\boldsymbol{\alpha}=\left\{\alpha_{1}, \ldots, \alpha_{m}\right\}$ collects the regularity of the basis functions at the internal knots, with $\alpha_{1}=\alpha_{m}=-1$ for the boundary knots, because of the open knot vector structure. Each basis function $B_{i}$ is non-negative and supported in the interval $\left[\xi_{i}, \xi_{i+p+1}\right]$. Moreover, these B-spline functions constitute a partition of unity, that is

$$
\sum_{i=1}^{n} B_{i}(x)=1 \quad \forall x \in(0,1) .
$$

The space of B-splines spanned by the basis functions $B_{i}$ will be denoted by

$$
S_{\boldsymbol{\alpha}}^{p}:=\operatorname{span}\left\{B_{i}\right\}_{i=1}^{n} \text {. }
$$

An example of quadratic B-splines constructed from the open knot vector

$$
\Xi=\{0,0,0,1 / 5,2 / 5,3 / 5,3 / 5,4 / 5,1,1,1\}
$$

is presented in Figure 1. In this case $\boldsymbol{\alpha}=\{-1,1,1,0,1,-1\}$. Notice that, since the knot $\xi_{6}=\xi_{7}=\zeta_{4}=3 / 5$ has multiplicity $r_{4}=2$, the fourth, fifth and sixth functions are only continuous $\left(\alpha_{4}=0\right)$ at that point.

When $\alpha_{i} \geq 0$, for all $2 \leq i \leq m-1$, the derivatives of functions in $S_{\boldsymbol{\alpha}}^{p}$ are splines as well. We have indeed

$$
\left\{\frac{d}{d x} v: v \in S_{\boldsymbol{\alpha}}^{p}\right\}=S_{\boldsymbol{\alpha}-1}^{p},
$$


where, here and in what follows, we adopt the notation $\boldsymbol{\alpha}-1=\left\{-1, \alpha_{2}-1, \ldots, \alpha_{m-1}-\right.$ $1,-1\}$, when $\boldsymbol{\alpha}=\left\{\alpha_{1}=-1, \alpha_{2}, \ldots, \alpha_{m-1}, \alpha_{m}=-1\right\}$

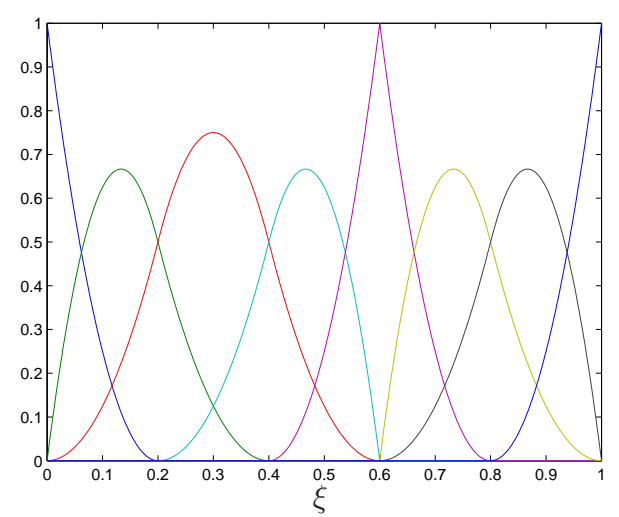

Figure 1: Quadratic B-splines basis functions constructed from the open knot vector $\Xi=$ $\{0,0,0,1 / 5,2 / 5,3 / 5,3 / 5,4 / 5,1,1,1\}$.

The space of piecewise smooth functions on $\left\{\zeta_{1}, \ldots, \zeta_{m}\right\}$, such that restricted to each subinterval $\left(\zeta_{i}, \zeta_{i+1}\right)$ admit a $C^{\infty}$ extension to the close interval $\left[\zeta_{i}, \zeta_{i+1}\right]$, and have $\alpha_{i}$ continuous derivative at $\zeta_{i}$, for all $i=2, \ldots, m-1$, is denoted by $\mathcal{C}_{\boldsymbol{\alpha}}^{\infty}$.

The previous definition of the B-splines space is extended to the two-dimensional framework in the following way. Let us consider the square $\widehat{\Omega}=(0,1)^{2} \subset \mathbb{R}^{2}$, which will be referred to as a patch. Given integers $p_{d}$ and $n_{d}$, with $d=1,2$, we introduce the knot vectors $\Xi_{d}=\left\{\xi_{1, d}, \xi_{2, d}, \ldots, \xi_{n_{d}+p_{d}+1, d}\right\}$ and the associated vectors $\left\{\zeta_{1, d}, \ldots, \zeta_{m_{d}, d}\right\}$, $\left\{r_{1, d}, \ldots, r_{m_{d}, d}\right\}$ and $\left\{\alpha_{1, d}, \ldots, \alpha_{m_{d}, d}\right\}$, as in the one-dimensional case. Associated with these knot vectors there is a mesh $Q_{h}$ of the patch, that is, a partition of $(0,1)^{2}$ into rectangles:

$$
Q_{h}=\left\{Q=\otimes_{d=1,2}\left(\zeta_{i_{d}, d}, \zeta_{i_{d}+1, d}\right), 1 \leq i_{d} \leq m_{d}-1\right\} .
$$

Given an element $Q \in Q_{h}$, we set by $h_{Q}=\operatorname{diam}(Q)$, while $h=\max \left\{h_{Q}, Q \in Q_{h}\right\}$ represents the global mesh size.

We associate to the two given knot vectors $\Xi_{d}, d=1,2$ the $p_{d}$-degree univariate B-splines basis functions $B_{i, d}$, with $i=1, \ldots, n_{d}$. Then, on the associated mesh $Q_{h}$, we define the tensor-product B-spline basis functions as

$$
B_{i j}:=B_{i, 1} \otimes B_{j, 2}, \quad i=1, \ldots, n_{1}, j=1, \ldots, n_{2} .
$$

Then, the tensor product B-spline space is defined as the space spanned by these basis functions, namely

$$
S_{\boldsymbol{\alpha}_{1}, \boldsymbol{\alpha}_{2}}^{p_{1}, p_{2}} \equiv S_{\boldsymbol{\alpha}_{1}, \boldsymbol{\alpha}_{2}}^{p_{1}, p_{2}}\left(Q_{h}\right):=S_{\boldsymbol{\alpha}_{1}}^{p_{1}} \otimes S_{\boldsymbol{\alpha}_{2}}^{p_{2}}=\operatorname{span}\left\{B_{i j}\right\}_{i=1, j=1}^{n_{1}, n_{2}} .
$$

Notice that the space $S_{\boldsymbol{\alpha}_{1}, \boldsymbol{\alpha}_{2}}^{p_{1}, p_{2}}\left(Q_{h}\right)$ is fully characterized by the mesh $Q_{h}$, by $p_{1}, p_{2}, \boldsymbol{\alpha}_{1}$ and $\boldsymbol{\alpha}_{2}$, as our notation reflects. The minimum regularity of the space is $\alpha:=\min \left\{\alpha_{i, d}: i=\right.$ $\left.2, \ldots, m_{d}-1, d=1,2\right\}$. This viewpoint is more natural in our context.

In a similar way, we define on $Q_{h}$ the space of piecewise smooth functions with interelement regularity assigned by the vectors $\boldsymbol{\alpha}_{1}$ and $\boldsymbol{\alpha}_{2}$, on the vertical and horizontal mesh 
edges, respectively. This is denoted by

$$
\mathcal{C}_{\boldsymbol{\alpha}_{1}, \boldsymbol{\alpha}_{2}}^{\infty}=\mathcal{C}_{\boldsymbol{\alpha}_{1}, \boldsymbol{\alpha}_{2}}^{\infty}\left(Q_{h}\right)=\mathcal{C}_{\boldsymbol{\alpha}_{1}}^{\infty} \otimes \mathcal{C}_{\boldsymbol{\alpha}_{2}}^{\infty} .
$$

Precisely, a function in $\mathcal{C}_{\boldsymbol{\alpha}_{1}, \boldsymbol{\alpha}_{2}}^{\infty}$ admits a $C^{\infty}$ extension in the closure of each element $Q \in \mathcal{Q}_{h}$, has $\alpha_{i, 1}$ continuous derivatives on the edges $\left\{\left(x_{1}, x_{2}\right): x_{1}=\zeta_{i, 1}, \zeta_{j, 2}<x_{2}<\zeta_{j+1,2}\right\}$, for $j=1, \ldots, m_{1}-1$, and $\alpha_{i, 2}$ continuous derivatives on the edges $\left\{\left(x_{1}, x_{2}\right): \zeta_{j, 1}<x_{1}<\right.$ $\left.\zeta_{j+1,1}, x_{2}=\zeta_{i, 2},\right\}$, for $j=1, \ldots, m_{1}-1$. From the definitions, $S_{\boldsymbol{\alpha}_{1}, \boldsymbol{\alpha}_{2}}^{p_{1}, p_{2}} \subset \mathcal{C}_{\boldsymbol{\alpha}_{1}, \boldsymbol{\alpha}_{2}}^{\infty}$.

Moreover, let $\mathcal{H}^{k}=\mathcal{H}^{k}\left(Q_{h}\right)$ be the broken $k$-order Sobolev space, that is, the space of functions in $L^{2}(\widehat{\Omega})$ whose restriction to $Q$ belongs to $H^{k}(Q)$, for all $Q \in Q_{h}$. The space $\mathcal{H}^{k}$ is endowed with seminorms and norm

$$
\begin{gathered}
|\cdot|_{\mathcal{H}^{l}}:=\left(\sum_{Q \in Q_{h}}|\cdot|_{H^{l}(Q)}^{2}\right)^{1 / 2}, \quad l=0,1, \ldots, k, \\
\|\cdot\|_{\mathcal{H}^{k}}:=\left(\sum_{Q \in Q_{h}}\|\cdot\|_{H^{k}(Q)}^{2}\right)^{1 / 2},
\end{gathered}
$$

Finally, the closure of $\mathcal{C}_{\boldsymbol{\alpha}_{1}, \boldsymbol{\alpha}_{2}}^{\infty}$ in $\mathcal{H}^{k}$ is denoted by $\mathcal{H}_{\boldsymbol{\alpha}_{1}, \boldsymbol{\alpha}_{2}}^{k}=\mathcal{H}_{\boldsymbol{\alpha}_{1}, \boldsymbol{\alpha}_{2}}^{k}\left(Q_{h}\right)$. The last space was introduced in [4] and named bent Sobolev space. Notice that the functions in $\mathcal{H}_{\boldsymbol{\alpha}_{1}, \boldsymbol{\alpha}_{2}}^{k}$ have derivatives up to order $\min \left\{k-1, \alpha_{i, d}\right\}$ that match, in the sense of traces, at the internal vertical $(d=1)$ or horizontal $(d=2)$ mesh edges lying on the line given by the equation $x_{d}=\zeta_{i, d}, i=2, \ldots, m_{d}-1$.

From an initial coarse mesh $Q_{h_{0}}$, refinements are constructed by knot insertion (with possible repetition, see [12]). Therefore, we end up considering a family of meshes $\left\{Q_{h}\right\}_{h \leq h_{0}}$ and associated spaces, with the global mesh size $h$ playing the role of family index, as usual in finite element literature.

We assume that our computational domain $\Omega \subset \mathbb{R}^{2}$ can be exactly parametrized by a geometrical mapping $\mathbf{F}: \widehat{\Omega} \longrightarrow \Omega$ which belongs to $\left(\mathcal{C}_{\gamma_{1}, \gamma_{2}}^{\infty}\left(Q_{h}\right)\right)^{2}$, with piecewise smooth inverse, and is independent of the mesh family index $h$. The global regularity of $\mathbf{F}$ is $\gamma:=\min \left\{\gamma_{i, d}: i=2, \ldots, m_{d}-1, d=1,2\right\}$. In our examples and numerical tests, $\mathbf{F}$ is taken in $\left(S_{\gamma_{0,1}, \gamma_{0,2}}^{q, q}\left(Q_{h_{0}}\right)\right)^{2}$, that is, $\mathbf{F}$ is a $q$-degree spline defined on the coarsest mesh $Q_{h_{0}}$ : to each of the B-spline basis functions $B_{i j}(\mathbf{x}) \in S_{\gamma_{0,1}, \gamma_{0,2}}^{q, q}\left(Q_{h_{0}}\right)$ is associated a control point $\mathbf{C}_{i j} \in \mathbb{R}^{2}$, such that

$$
\mathbf{x} \longmapsto \mathbf{F}(\mathbf{x}):=\sum_{i j} B_{i j}(\mathbf{x}) \mathbf{C}_{i j}
$$

\subsection{The De Rham diagram}

For any function $w \in H^{1}(\Omega)$ it holds that $\operatorname{curl} \operatorname{grad} w=0$, thus it is clear that $\operatorname{grad} w \in$ $\mathbf{H}(\operatorname{curl} ; \Omega)$. Moreover, since the domain $\Omega$ is simply connected, we also know that the range of the gradient operator is equal to the kernel of the curl operator, namely $\operatorname{Im}(\operatorname{grad})=$ $\operatorname{ker}(\operatorname{curl})$. This is summarized in the De Rham diagram:

$$
\mathbb{R} \longrightarrow H^{1}(\Omega) \stackrel{\text { grad }}{\longrightarrow} \mathbf{H}(\operatorname{curl} ; \Omega) \stackrel{\text { curl }}{\longrightarrow} L^{2}(\Omega) \longrightarrow 0 .
$$

In order to discretize the continuous problems of Section 2 it is necessary to construct finite dimensional spaces $\mathrm{U}_{h} \subset H^{1}(\Omega), \mathrm{V}_{h} \subset \mathbf{H}(\operatorname{curl} ; \Omega)$ and $\mathrm{W}_{h} \subset L^{2}(\Omega)$, maintaining the 
same relationships of the continuous spaces given in (13). This is in fact what one does when using edge finite elements to approximate $\mathbf{H}(\operatorname{curl} ; \Omega)$, and standard Lagrange finite elements of suitable degree to discretize $H^{1}(\Omega)$ and $L^{2}(\Omega)$ (see [18]). The first step is to construct suitable discretizations of the spaces in the patch $\widehat{\Omega}$. With the notation of Section 3.1 , let $Q_{h}$ be a mesh and $S_{\boldsymbol{\alpha}_{1}, \boldsymbol{\alpha}_{2}}^{p_{1}, p_{2}}\left(Q_{h}\right)$ the associated space of splines of order $p_{1}$ in the $x$ direction, $p_{2}$ in the $y$ direction, and smoothness given by the vectors $\boldsymbol{\alpha}_{1}, \boldsymbol{\alpha}_{2}$. We assume, from now on, that $\alpha=\min \left\{\alpha_{i, d}: i=2, \ldots, m_{d}-1, d=1,2\right\}$ is nonnegative.

Recalling (8), the gradients of $S_{\boldsymbol{\alpha}_{1}, \boldsymbol{\alpha}_{2}}^{p_{1}, p_{2}}\left(Q_{h}\right)$ belong to $S_{\boldsymbol{\alpha}_{1}-1, \boldsymbol{\alpha}_{2}}^{p_{1}-1, p_{2}}\left(Q_{h}\right) \times S_{\boldsymbol{\alpha}_{1}, \boldsymbol{\alpha}_{2}-1}^{p_{1}, p_{2}-1}\left(Q_{h}\right)$, and the curl of fields belonging to this last space is in $S_{\boldsymbol{\alpha}_{1}-1, \boldsymbol{\alpha}_{2}-1}^{p_{1}-1, p_{2}-1}\left(Q_{h}\right)$. Notice that these spaces are built from different knot vectors, though the difference is only in the multiplicity of the boundary knots. ${ }^{1}$ The following proposition states that the previous discrete spaces form a De Rham diagram on the patch $\widehat{\Omega}$.

Proposition 3.1. With the notation and assumptions above, the following diagram holds

$$
\mathbb{R} \rightarrow S_{\boldsymbol{\alpha}_{1}, \boldsymbol{\alpha}_{2}}^{p_{1}, p_{2}}\left(Q_{h}\right) \stackrel{\text { grad }}{\longrightarrow} S_{\boldsymbol{\alpha}_{1}-1, \boldsymbol{\alpha}_{2}}^{p_{1}-1, p_{2}}\left(Q_{h}\right) \times S_{\boldsymbol{\alpha}_{1}, \boldsymbol{\alpha}_{2}-1}^{p_{1}, p_{2}-1}\left(Q_{h}\right) \stackrel{\text { curl }}{\longrightarrow} S_{\boldsymbol{\alpha}_{1}-1, \boldsymbol{\alpha}_{2}-1}^{p_{1}-1, p_{2}-1}\left(Q_{h}\right) \rightarrow 0 .
$$

Proof. We already observed that, from the definition of the gradient and the curl operators, for any functions $w \in S_{\boldsymbol{\alpha}_{1}, \boldsymbol{\alpha}_{2}}^{p_{1}, p_{2}}\left(Q_{h}\right)$ and $\mathbf{u} \in S_{\boldsymbol{\alpha}_{1}-1, \boldsymbol{\alpha}_{2}}^{p_{1}-1, p_{2}}\left(Q_{h}\right) \times S_{\boldsymbol{\alpha}_{1}, \boldsymbol{\alpha}_{2}-1}^{p_{1}, p_{2}-1}\left(Q_{h}\right)$ we have $\operatorname{grad} w \in$ $S_{\boldsymbol{\alpha}_{1}-1, \boldsymbol{\alpha}_{2}}^{p_{1}-1, p_{2}}\left(Q_{h}\right) \times S_{\boldsymbol{\alpha}_{1}, \boldsymbol{\alpha}_{2}-1}^{p_{1}, p_{2}-1}\left(Q_{h}\right)$ and curl $\mathbf{u} \in S_{\boldsymbol{\alpha}_{1}-1, \boldsymbol{\alpha}_{2}-1}^{p_{1}-1, p_{2}-1}\left(Q_{h}\right)$. Moreover, it is also clear that $\operatorname{curl}(\operatorname{grad} w)=0$, which means that

$$
\operatorname{grad}\left(S_{\boldsymbol{\alpha}_{1}, \boldsymbol{\alpha}_{2}}^{p_{1}, p_{2}}\left(\mathcal{Q}_{h}\right)\right) \subset \operatorname{ker}(\operatorname{curl}) \cap\left\{S_{\boldsymbol{\alpha}_{1}-1, \boldsymbol{\alpha}_{2}}^{p_{1}-1, p_{2}}\left(\mathcal{Q}_{h}\right) \times S_{\boldsymbol{\alpha}_{1}, \boldsymbol{\alpha}_{2}-1}^{p_{1}, p_{2}-1}\left(\mathcal{Q}_{h}\right)\right\}
$$

Therefore, it is sufficient to prove that both spaces have the same dimension. The dimension of $S_{\boldsymbol{\alpha}_{1}, \boldsymbol{\alpha}_{2}}^{p_{1}, p_{2}}\left(Q_{h}\right)$ is equal to $n_{1} n_{2}$, with the notation above, and, since $\operatorname{dim}(\operatorname{ker}(\operatorname{grad}))=1$, we infer that $\operatorname{dim}\left(\operatorname{grad}\left(S_{\boldsymbol{\alpha}_{1}, \boldsymbol{\alpha}_{2}}^{p_{1}, p_{2}}\left(Q_{h}\right)\right)\right)=n_{1} n_{2}-1$. For the kernel of the curl operator we know that

$$
\begin{aligned}
\operatorname{dim}(\operatorname{ker}(\operatorname{curl}))+\operatorname{dim}(\operatorname{Im}(\operatorname{curl})) & =\operatorname{dim}\left(S_{\boldsymbol{\alpha}_{1}-1, \boldsymbol{\alpha}_{2}}^{p_{1}-1, p_{2}}\left(\mathcal{Q}_{h}\right) \times S_{\boldsymbol{\alpha}_{1}, \boldsymbol{\alpha}_{2}-1}^{p_{1}, p_{2}-1}\left(\mathcal{Q}_{h}\right)\right) \\
& =\left(n_{1}-1\right) n_{2}+n_{1}\left(n_{2}-1\right) \\
& =2 n_{1} n_{2}-n_{1}-n_{2},
\end{aligned}
$$

where the last equality holds from the definition of the space. Thus, the result will be proved if we can show that $\operatorname{dim}(\operatorname{Im}(\operatorname{curl}))=\left(n_{1}-1\right)\left(n_{2}-1\right)=n_{1} n_{2}-n_{1}-n_{2}+1$. Since this is the dimension of the space $S_{\boldsymbol{\alpha}_{1}-1, \boldsymbol{\alpha}_{2}-1}^{p_{1}-1, p_{2}-1}\left(Q_{h}\right)$, we have to prove that the curl operator is surjective. This is easily seen from the properties of the derivative. We recall (8), that is, in one space

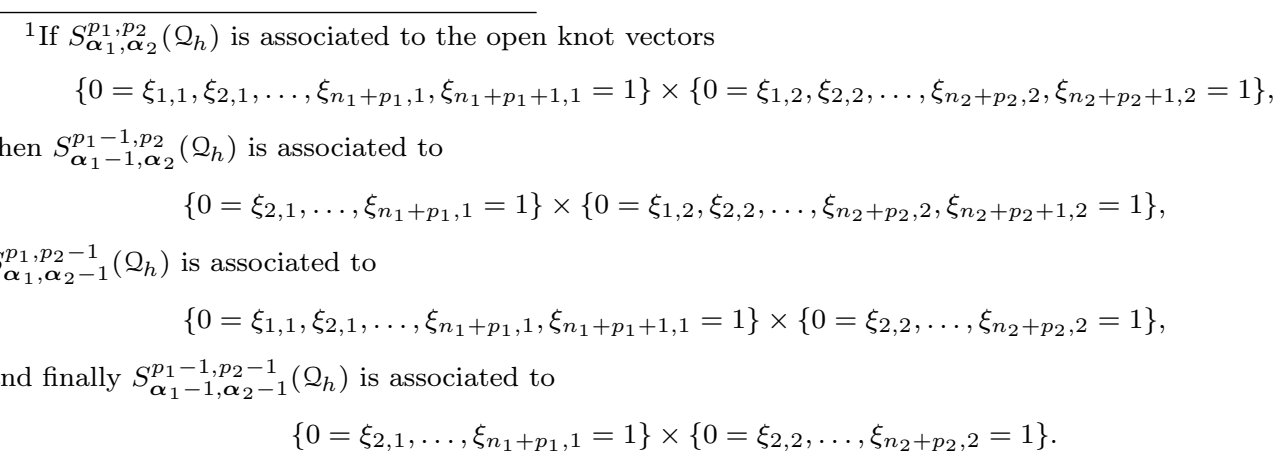


dimension the derivative operator maps the $n_{1}$-dimensional space $S_{\boldsymbol{\alpha}_{1}}^{p_{1}}$ into the $\left(n_{1}-1\right)$ dimensional space $S_{\boldsymbol{\alpha}_{1}-1}^{p_{1}-1}$, and since its kernel has dimension equal to one, the derivative operator is surjective. Analogously, the first partial derivative is a surjective operator from $S_{\boldsymbol{\alpha}_{1}, \boldsymbol{\alpha}_{2}-1}^{p_{1}, p_{2}-1}\left(Q_{h}\right)$ into $S_{\boldsymbol{\alpha}_{1}-1, \boldsymbol{\alpha}_{2}-1}^{p_{1}-p_{2}-1}\left(Q_{h}\right)$, and as an immediate consequence of its definition, the curl operator is also surjective from $S_{\boldsymbol{\alpha}_{1}-1, \boldsymbol{\alpha}_{2}}^{p_{1}-1, p_{2}}\left(Q_{h}\right) \times S_{\boldsymbol{\alpha}_{1}, \boldsymbol{\alpha}_{2}-1}^{p_{1}, p_{2}-1}\left(Q_{h}\right)$ into $S_{\boldsymbol{\alpha}_{1}-1, \boldsymbol{\alpha}_{2}-1}^{p_{1}-1, p_{2}-1}\left(Q_{h}\right)$.

Once we have defined the finite dimensional spaces in the patch $\widehat{\Omega}$, the next step is to construct the corresponding spaces in our physical domain $\Omega$. The finite dimensional spaces for the scalar functions are defined via the geometrical parametrization $\mathbf{F}: \widehat{\Omega} \longrightarrow \Omega$ as follows:

$$
\begin{aligned}
\mathrm{U}_{h} & =\left\{\phi: \phi \circ \mathbf{F}=\widehat{\phi}, \widehat{\phi} \in S_{\boldsymbol{\alpha}_{1}, \boldsymbol{\alpha}_{2}}^{p_{1}, p_{2}}\left(Q_{h}\right)\right\} \\
\mathrm{W}_{h} & =\left\{w: w \circ \mathbf{F}=\frac{1}{\operatorname{det}(D \mathbf{F})} \widehat{w}, \widehat{w} \in S_{\boldsymbol{\alpha}_{1}-1, \boldsymbol{\alpha}_{2}-1}^{p_{1}-1, p_{2}-1}\left(Q_{h}\right)\right\} .
\end{aligned}
$$

where $D \mathbf{F}$ is the Jacobian matrix of the parametrization $\mathbf{F}$. In order to conserve the curl properties, vector functions in the patch are mapped into the physical domain via a covariant transformation, hence the corresponding finite dimensional space is defined as:

$$
\mathrm{V}_{h}=\left\{\mathbf{u}: \mathbf{u} \circ \mathbf{F}=(D \mathbf{F})^{-\top} \widehat{\mathbf{u}}, \widehat{\mathbf{u}} \in S_{\boldsymbol{\alpha}_{1}-1, \boldsymbol{\alpha}_{2}}^{p_{1}-1, p_{2}}\left(Q_{h}\right) \times S_{\boldsymbol{\alpha}_{1}, \boldsymbol{\alpha}_{2}-1}^{p_{1}, p_{2}-1}\left(Q_{h}\right)\right\},
$$

The following proposition states that the De Rham diagram also holds in the finite dimensional spaces defined in the physical domain $\Omega$.

Proposition 3.2. Let the spaces $\mathrm{U}_{h}, \mathrm{~V}_{h}$ and $\mathrm{W}_{h}$ be defined as in (17)-(19). Then the following diagram holds

$$
\mathbb{R} \rightarrow \mathrm{U}_{h} \stackrel{\text { grad }}{\longrightarrow} \mathrm{V}_{h} \stackrel{\text { curl }}{\longrightarrow} \mathrm{W}_{h} \rightarrow 0
$$

Proof. The result is a direct consequence of Proposition 3.1, and the fact that the space $\mathrm{V}_{h}$ is defined via a curl conserving transformation, as it is explained in [18, Sect. 3.9].

Remark 3.1. We notice that, leaving the parametrization aside, that is, working on the patch $\widehat{\Omega}$, and assuming that $p_{1}=p_{2}=p$, our discretization scheme with $\boldsymbol{\alpha}_{1}=\{-1,0, \ldots, 0,-1\}$ and $\boldsymbol{\alpha}_{2}=\{-1,0, \ldots, 0,-1\}$ is equivalent to Nédélec's finite elements of the first kind. As it is known, in this case the normal component of the approximation is not necessarily continuous. One of the advantages of our method is that the regularity of the solution can be increased with respect to edge finite elements. Indeed, our method provides an approximated solution with up to $p-2$ continuous derivatives. In particular, by using quadratic elements it is possible to find a globally continuous numerical solution, with well defined divergence in the space $L^{2}(\Omega)$.

\subsection{The discrete problem}

Now that we have constructed the finite dimensional spaces in the physical domain $\Omega$, we can introduce the discrete version of our problem. In order to do so, we must define some spaces satisfying certain boundary conditions. As it is explained in [16], natural boundary conditions are imposed as in standard finite element analysis. To impose Dirichlet boundary conditions on the boundary $\Gamma_{D} \subseteq \partial \Omega$, let us introduce the notation $\gamma_{D}=\mathbf{F}^{-1}\left(\Gamma_{D}\right)$. First we define the constrained space in the patch $\widehat{\Omega}$

$$
V_{h, \gamma_{D}}:=\left(S_{\boldsymbol{\alpha}_{1}-1, \boldsymbol{\alpha}_{2}}^{p_{1}-1, p_{2}}\left(Q_{h}\right) \times S_{\boldsymbol{\alpha}_{1}, \boldsymbol{\alpha}_{2}-1}^{p_{1}, p_{2}-1}\left(Q_{h}\right)\right) \cap \mathbf{H}_{0 ; \gamma_{D}}(\operatorname{curl} ; \widehat{\Omega}) .
$$


Analogously, we can define the constrained space in the physical domain $\Omega$

$$
\bigvee_{h ; \Gamma_{D}}:=\bigvee_{h} \cap \mathbf{H}_{0 ; \Gamma_{D}}(\operatorname{curl} ; \Omega)=\left\{\mathbf{u}: \mathbf{u} \circ \mathbf{F}=(D \mathbf{F})^{-\top} \widehat{\mathbf{u}}, \widehat{\mathbf{u}} \in V_{h, \gamma_{D}}\right\},
$$

and in the particular case of $\Gamma_{D}=\partial \Omega$, we denote it by $\mathrm{V}_{h ; 0} \equiv \mathrm{V}_{h ; \partial \Omega}:=\mathrm{V}_{h} \cap \mathbf{H}_{0}(\operatorname{curl} ; \Omega)$.

Once we have introduced the constrained spaces it is possible to write the discrete version of our problems. The discrete eigenvalue problem reads:

Find $\omega \in \mathbb{R}$ and $\mathbf{u}_{h} \in \mathrm{V}_{h ; 0}, \mathbf{u}_{h} \neq \mathbf{0}$, satisfying

$$
\left(\operatorname{curl} \mathbf{u}_{h}, \operatorname{curl} \mathbf{v}_{h}\right)=\omega^{2}\left(\mathbf{u}_{h}, \mathbf{v}_{h}\right) \quad \forall \mathbf{v}_{h} \in \mathrm{V}_{h ; 0} .
$$

And the discrete version of the source problem (4) is the following:

Given $\mathbf{f} \in \mathbf{L}^{2}(\Omega)$, find $\mathbf{u}_{h} \in \mathrm{V}_{h ; \Gamma_{D}}$ such that

$$
\int_{\Omega} \operatorname{curl} \mathbf{u}_{h} \operatorname{curl} \mathbf{v}_{h}+\int_{\Omega} \mathbf{u}_{h} \cdot \mathbf{v}_{h}=\int_{\Omega} \mathbf{f} \cdot \mathbf{v}_{h} \quad \forall \mathbf{v}_{h} \in \mathrm{V}_{h ; \Gamma_{D}} .
$$

Remark 3.2. It is also possible to define constrained spaces in $\mathrm{U}_{h}$ and $\mathrm{W}_{h}$ in order to obtain the De Rham diagram in the constrained spaces. For example, we can define $\mathrm{U}_{h ; 0}:=$ $\mathrm{U}_{h} \cap H_{0}^{1}(\Omega)$, which following the notation of Proposition 3.1 has dimension equal to $\left(n_{1}-\right.$ $2)\left(n_{2}-2\right)$. Then it can be seen that $\operatorname{ker}(\operatorname{curl}) \cap \mathrm{V}_{h ; 0}=\operatorname{grad}\left(\mathrm{U}_{h ; 0}\right)$ and the dimension of this space is also equal to $\left(n_{1}-2\right)\left(n_{2}-2\right)$.

\section{Approximation analysis}

In this section we present an error analysis which is sub-optimal and valid only for regular solutions of the problem (24). Indeed, this should be considered as the starting point for a more general analysis able to cover also the case of non-regular solutions and the eigenvalue problem (23). The reason why we cannot perform a general analysis is that we do not have suitable commuting projectors at our disposal. On the other hand, we treat in optimal way the presence and the regularity of the mapping $\mathbf{F}$.

The present analysis follows [4] and use results in [21]. Since the problem (24) is coercive, the analysis of the error of the numerical method is reduced to the analysis of the best approximation error in the norm $\mathbf{H}(\operatorname{curl}, \Omega)$.

In order to present our analysis, we recall and specify some notation. We assume, for the sake of simplicity, that the same degree of approximation $p=p_{1}=p_{2}$ is adopted in both Cartesian directions. Given the mesh $Q_{h}$, which is assumed to be shape regular (that is, the element length ratios are assumed to be bounded uniformly with respect to $h$ ) and the vectors $\boldsymbol{\alpha}_{1}, \boldsymbol{\alpha}_{2}$, the discrete spaces are constructed as detailed in previous sections. We recall that $\alpha:=\min \left\{\alpha_{i, d}: i=2, \ldots, m_{d}-1, d=1,2\right\}$. The geometrical mapping $\mathbf{F}: \widehat{\Omega} \longrightarrow \Omega$ belongs to $\left(\mathcal{C}_{\gamma_{1}, \gamma_{2}}^{\infty}\left(Q_{h}\right)\right)^{2}$, for some given vectors $\gamma_{1}$ and $\gamma_{2}$, with the scalar $\gamma=\min \left\{\gamma_{i, d}: i=2, \ldots, m_{d}-1, d=1,2\right\}$ denoting its global regularity, which we assume nonnegative. Furthermore, $\mathbf{F}$ has a piecewise smooth inverse, and, since it is defined on the coarsest level of the mesh, is independent of the mesh family index $h$.

The following approximation result on the parametric space $\widehat{\Omega}$ follows from $[4$, Lemma 3.3].

Lemma 4.1. There exists a projector $\Pi_{S}: \mathbf{L}^{2}(\widehat{\Omega}) \rightarrow S_{\boldsymbol{\alpha}_{1}-1, \boldsymbol{\alpha}_{2}}^{p-1, p}\left(Q_{h}\right) \times S_{\boldsymbol{\alpha}_{1}, \boldsymbol{\alpha}_{2}-1}^{p, p-1}\left(Q_{h}\right)$, such that, for all $\widehat{\mathbf{u}} \in \mathcal{H}_{\boldsymbol{\alpha}_{1}-1, \boldsymbol{\alpha}_{2}}^{l}\left(Q_{h}\right) \times \mathcal{H}_{\boldsymbol{\alpha}_{1}, \boldsymbol{\alpha}_{2}-1}^{l}\left(Q_{h}\right)$ it holds:

$$
\sum_{Q \in Q_{h}}\left|\widehat{\mathbf{u}}-\Pi_{S} \widehat{\mathbf{u}}\right|_{\mathbf{H}^{k}(Q)}^{2} \leq C \sum_{Q \in Q_{h}} h_{Q}^{2(l-k)}|\widehat{\mathbf{u}}|_{\mathbf{H}^{l}(Q)}^{2}
$$


for any $0 \leq k \leq l \leq p$, and with $C$ only dependent on $p$.

From Lemma 4.1, we derive the approximation error estimates on the physical domain. We define the projector onto $\mathrm{V}_{h}$ by push-forward:

$$
\left(\Pi_{\vee_{h}} \mathbf{u}\right) \circ \mathbf{F}=D \mathbf{F}^{-T} \Pi_{S}(\widehat{\mathbf{u}}), \quad \widehat{\mathbf{u}}=D \mathbf{F}^{T}(\mathbf{u} \circ \mathbf{F}) .
$$

The projector $\Pi_{V_{h}}$ verifies the following approximation properties.

Proposition 4.1. With the previous notation and assumptions, let $\alpha \geq 0,1 \leq l \leq p$.

If $\gamma_{d} \geq \boldsymbol{\alpha}_{d}$ (that is, $\left.\gamma_{i, d} \geq \alpha_{i, d}, \forall i\right)$, for $d=1,2$. Then, there exists a constant $C=C(\mathbf{F}, p)$ such that

$$
\left\|\mathbf{u}-\Pi_{\mathrm{V}_{h}} \mathbf{u}\right\|_{\mathbf{L}^{2}(\Omega)}+h\left\|\operatorname{curl}\left(\mathbf{u}-\Pi_{\mathrm{V}_{h}} \mathbf{u}\right)\right\|_{L^{2}(\Omega)} \leq C h^{l}\|\mathbf{u}\|_{\mathbf{H}^{l}(\Omega)}, \quad \forall \mathbf{u} \in \mathbf{H}^{l}(\Omega) .
$$

Otherwise, for any $1 \leq \eta<\gamma+1 / 2$ there exists a constant $C=C(\mathbf{F}, \gamma, p)$ such that

$$
\left\|\mathbf{u}-\Pi_{\mathbb{V}_{h}} \mathbf{u}\right\|_{\mathbf{L}^{2}(\Omega)}+h\left\|\operatorname{curl}\left(\mathbf{u}-\Pi_{\mathbb{V}_{h}} \mathbf{u}\right)\right\|_{L^{2}(\Omega)} \leq C h^{\eta}\|\mathbf{u}\|_{\mathbf{H}^{\eta}(\Omega)}, \quad \forall \mathbf{u} \in \mathbf{H}^{\eta}(\Omega) .
$$

Proof. Let $K=F(Q)$, for a generic $Q \in Q_{h}$. We can compute:

$$
\begin{aligned}
\int_{K}\left|\mathbf{u}-\Pi_{\mathrm{V}_{h}} \mathbf{u}\right|^{2} & =\int_{Q}|\operatorname{det} D \mathbf{F}|\left|\mathbf{u} \circ \mathbf{F}-\left(\Pi_{\mathrm{V}_{h}} \mathbf{u}\right) \circ \mathbf{F}\right|^{2} \\
& \leq\|\operatorname{det} D \mathbf{F}\|_{L^{\infty}(Q)}\left|\left\|D \mathbf{F}^{-T}\right\|_{\mathbf{L}^{\infty}(K)} \int_{Q}\right| \widehat{\mathbf{u}}-\left.\Pi_{S} \widehat{\mathbf{u}}\right|^{2} .
\end{aligned}
$$

Then

$$
\left\|\mathbf{u}-\Pi_{\mathrm{V}_{h}} \mathbf{u}\right\|_{\mathbf{L}^{2}(\Omega)} \leq C(\mathbf{F})\left\|\widehat{\mathbf{u}}-\Pi_{S} \widehat{\mathbf{u}}\right\|_{\mathbf{L}^{2}(\widehat{\Omega})} .
$$

Consider the first component of $\widehat{\mathbf{u}}$, which we denote $\widehat{u}_{1}$; from the definition (26)

$$
\widehat{u}_{1}\left(x_{1}, x_{2}\right)=\frac{\partial \mathbf{F}}{\partial x_{1}}\left(x_{1}, x_{2}\right) \cdot(\mathbf{u} \circ \mathbf{F})\left(x_{1}, x_{2}\right) .
$$

From the assumptions on $\mathbf{u}$ and $\mathbf{F}$, one has $\frac{\partial \mathbf{F}}{\partial x_{1}} \in\left(\mathcal{C}_{\boldsymbol{\gamma}_{1}-1, \boldsymbol{\gamma}_{2}}^{\infty}\left(Q_{h}\right)\right)^{2}$, and $\mathbf{u} \circ \mathbf{F} \in$ $\mathcal{H}_{\boldsymbol{\gamma}_{1}, \boldsymbol{\gamma}_{2}}^{l}\left(Q_{h}\right)$. Therefore, we obtain $\widehat{u}_{1} \in \mathcal{H}_{\boldsymbol{\gamma}_{1}-1, \boldsymbol{\gamma}_{2}}^{l}\left(Q_{h}\right) \subseteq \mathcal{H}_{\boldsymbol{\alpha}_{1}-1, \boldsymbol{\alpha}_{2}}^{l}\left(Q_{h}\right)$, using $\boldsymbol{\gamma}_{d} \geq \boldsymbol{\alpha}_{d}$. In an analogous way, for the second component of $\widehat{\mathbf{u}}$, denoted $\widehat{u}_{2}$, we can prove $\widehat{u}_{2} \in$ $\mathcal{H}_{\boldsymbol{\alpha}_{1}, \boldsymbol{\alpha}_{2}-1}^{l}\left(Q_{h}\right)$. Therefore $\widehat{\mathbf{u}} \in \mathcal{H}_{\boldsymbol{\alpha}_{1}-1, \boldsymbol{\alpha}_{2}}^{l}\left(Q_{h}\right) \times \mathcal{H}_{\boldsymbol{\alpha}_{1}, \boldsymbol{\alpha}_{2}-1}^{l}\left(Q_{h}\right)$. We also have, for the smoothness of $\mathbf{F}$ within each element, by change of variable

$$
\sum_{Q \in Q_{h}}|\widehat{\mathbf{u}}|_{\mathbf{H}^{l}(Q)}^{2} \leq C(\mathbf{F})\|\mathbf{u}\|_{\mathbf{H}^{l}(\Omega)}^{2} .
$$

We can then use (25), with (29) and (31), and obtain

$$
\left\|\mathbf{u}-\Pi_{\mathrm{V}_{h}} \mathbf{u}\right\|_{\mathbf{L}^{2}(\Omega)} \leq C(\mathbf{F}) h^{l}\|\mathbf{u}\|_{\mathbf{H}^{l}(\Omega)} .
$$

For the estimate of the term $\left\|\operatorname{curl} \mathbf{u}-\operatorname{curl} \Pi_{V_{h}} \mathbf{u}\right\|_{L^{2}(\Omega)}$ we recall that

$$
\begin{gathered}
(\operatorname{curl} \mathbf{u}) \circ \mathbf{F}=\frac{1}{\operatorname{det} D \mathbf{F}} \operatorname{curl} \widehat{\mathbf{u}} . \\
\left(\operatorname{curl} \Pi_{\vee_{h}} \mathbf{u}\right) \circ \mathbf{F}=\frac{1}{\operatorname{det} D \mathbf{F}} \operatorname{curl} \Pi_{S} \widehat{\mathbf{u}} .
\end{gathered}
$$


Therefore, by change of variable,

$$
\begin{aligned}
\int_{K}\left|\operatorname{curl} \mathbf{u}-\operatorname{curl} \Pi_{\mathrm{V}_{h}} \mathbf{u}\right|^{2} & \leq\|\operatorname{det} D \mathbf{F}\|_{L^{\infty}(Q)}^{-1} \int_{Q}\left|\operatorname{curl} \widehat{\mathbf{u}}-\operatorname{curl} \Pi_{S} \widehat{\mathbf{u}}\right|^{2} \\
& \leq\|\operatorname{det} D \mathbf{F}\|_{L^{\infty}(Q)}^{-1}\left|\widehat{\mathbf{u}}-\Pi_{S} \widehat{\mathbf{u}}\right|_{\mathbf{H}^{1}(Q)}^{2} .
\end{aligned}
$$

Reasoning as before, one obtains

$$
\left\|\operatorname{curl} \mathbf{u}-\operatorname{curl} \Pi_{\mathrm{V}_{h}} \mathbf{u}\right\|_{L^{2}(\Omega)} \leq C(\mathbf{F}) h^{l-1}\|\mathbf{u}\|_{\mathbf{H}^{l}(\Omega)} .
$$

If the assumption $\gamma_{i, d} \geq \alpha_{i, d}$ does not hold true for all $i$, and $d=1,2$, then, in general, the vector field $\widehat{\mathbf{u}} \notin \mathcal{H}_{\boldsymbol{\alpha}_{1}-1, \boldsymbol{\alpha}_{2}}^{l}\left(Q_{h}\right) \times \mathcal{H}_{\boldsymbol{\alpha}_{1}, \boldsymbol{\alpha}_{2}-1}^{l}\left(Q_{h}\right)$ and Lemma 4.1 cannot be applied. Instead, by standard Sobolev embedding theorem, we know that $\widehat{\mathbf{u}} \in \mathbf{H}^{\eta}(\Omega)$ for all $\eta<\gamma+1 / 2$ and we can apply a standard Bramble-Hilbert Lemma. Since $\Pi_{S}$ reproduces polynomials up to degree $p-1$, and $1 \leq \eta \leq p$, by usual scaling argument we obtain

$$
\left\|\mathbf{u}-\Pi_{\mathrm{V}_{h}} \mathbf{u}\right\|_{\mathbf{L}^{2}(\Omega)}+h\left|\mathbf{u}-\Pi_{\mathrm{V}_{h}} \mathbf{u}\right|_{\mathbf{H}^{1}(\Omega)}^{2} \cdot \leq C(\mathbf{F}) h^{\eta}\|\mathbf{u}\|_{\mathbf{H}^{\eta}(\Omega)},
$$

eventually giving (28).

Remark 4.1. The Proposition 4.1 says that, in order to have an optimal best approximation error on regular vector fields, the parametrization $\mathbf{F}$ for the physical domain must be pointwise at least as regular as the basis functions we then use to construct test and trial vector fields. This fact is clearly demonstrated by our numerical results.

\section{$5 \quad$ Numerical tests}

In this section we present the results given by our discretization technique when applied to three different benchmark problems. The first one is the computation of Maxwell's eigenvalues in a square. In the second test we solve the same problem in a non-convex L-shaped domain. Finally, the third one is a source problem posed in the same L-shaped domain, and the solution is known to have an unbounded singularity in the non-convex corner. The numerical method has been implemented in MATLAB, using the algorithms given in [20] for the evaluation of B-splines and their derivatives.

\subsection{Maxwell's eigenvalues in the square}

In this first test we numerically solve problem (2) in the square $(0, \pi)^{2} \subset \mathbb{R}^{2}$. The eigenvalues are given by $\omega^{2}=i^{2}+j^{2}$, with $i, j=0,1, \ldots$, and their corresponding eigenfunctions are $\mathbf{u}=(-j \cos (i x) \sin (j y), i \sin (i x) \cos (j y))$.

The geometry is described using four parameterizations, which differ in their degrees and continuities. The coarsest mesh is built from the open knot vectors $\Xi_{1}=\Xi_{2}=$ $\left\{0, \ldots 0, \frac{1}{4}, \frac{2}{4}, \frac{3}{4}, 1, \ldots, 1\right\}$. Then we construct the spaces of B-splines $S_{\gamma_{1}, \gamma_{2}}^{q, q}\left(Q_{h 0}\right)$, with $q=1, \ldots, 4$, and $\gamma_{1}=\gamma_{2}=\{-1, \gamma, \gamma, \gamma,-1\}$, with $\gamma=q-1$. The parametrization is then defined with a uniform distribution of the control points $\mathbf{C}_{i j} \in[0, \pi]^{2}$, for $i, j=1, \ldots, q+4$. This choice of the control points yields a $C^{\infty}$ parametrization for $q=1$ (in fact, the product of $\pi$ by the identity), and $C^{\gamma}$ parameterizations for $q=2,3,4$.

To construct the spaces of trial and test functions we consider the previous knot vectors with different multiplicities of the internal knots $\left\{\frac{1}{4}, \frac{2}{4}, \frac{3}{4}\right\}$. In particular, we consider B-splines of degree $p=2,3,4$, and global regularity $\alpha<p$. That is, we require for the 
internal knots a multiplicity $p-\alpha$, to construct the spaces $S_{\boldsymbol{\alpha}_{1}-1, \boldsymbol{\alpha}_{2}}^{p-1, p}\left(Q_{h 0}\right) \times S_{\boldsymbol{\alpha}_{1}, \boldsymbol{\alpha}_{2}-1}^{p, p-1}\left(Q_{h 0}\right)$, $\boldsymbol{\alpha}_{1}=\boldsymbol{\alpha}_{2}=\{-1, \alpha, \alpha, \alpha,-1\}$. To each space in the parametric domain we apply the covariant transformation described in Section 3.2, with the four parametrizations described above. This means that we have several discretization spaces $\mathrm{V}_{h ; 0} \equiv \mathrm{V}_{h ; 0}(p, \alpha, \mathbf{F})$ depending on the degree and the continuity of the B-splines basis functions, but also on the parametrization of the physical domain. The problem is then solved in five successively refined meshes, where we refine using knot insertion (see [16]) of knots with multiplicity 1, and maintaining the previous $p-\alpha$ multiplicity of the initial internal knots $\frac{1}{4}, \frac{2}{4}, \frac{3}{4}$. This is named $k$-refinement in $[16]$.

In the figures we present the convergence rate of the approximation to the eigenfunction corresponding to the pair $(i, j)=(3,3)$ in several cases. In Figure 2 the $C^{\infty}$ and $C^{3}$ parameterizations are considered, and the convergence rate is seen to be always equal to $p$. In Figure 3 we show the convergence results for $p=4$ and different values of $\alpha$, when considering the $C^{1}$ and $C^{2}$ parametrizations. As was pointed in Remark 4.1, the optimal convergence rate is only achieved when the regularity of the test functions is lower or equal than the regularity of the parametrization.
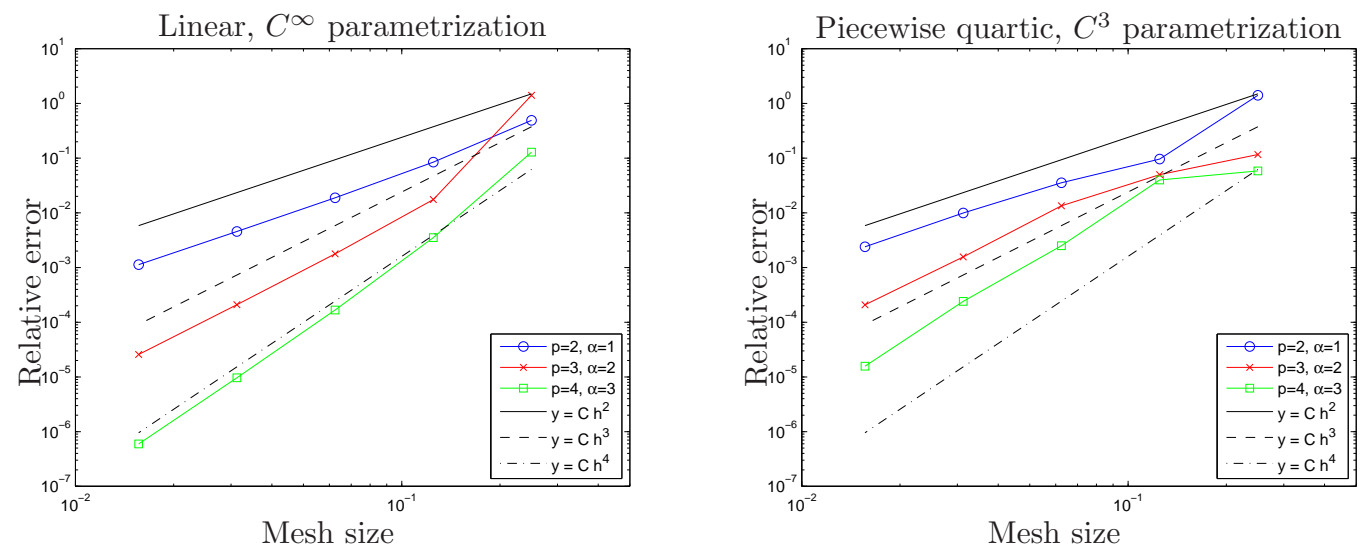

Figure 2: Errors on the $(3,3)$ eigenfunction, in the energy norm on the square. Linear and piecewise quartic parameterizations.

In Table 1 we show the first non-null eigenvalues computed in the five meshes when considering $p=2, \alpha=1$ and the linear $C^{\infty}$ parametrization. As can be seen our results are free of spurious modes. Moreover, following the notation of Proposition 3.1, the dimension of the space $\mathrm{V}_{h ; 0}$, that is, the number of degrees of freedom, is equal to $2(n-1)(n-2)$, and the number of computed zeros is equal to $(n-2)^{2}$. This last value is exactly the dimension of the kernel of the curl operator, as already explained in Remark 3.2.

As we noticed in Remark 3.1, for $\alpha \geq 1$ the solution of the problem is approximated with continuous piecewise polynomials. As a consequence the divergence of our numerical solution is well defined and can be numerically computed. To show this we have considered the approximation of the eigenfunction corresponding to the pair $(m, n)=(3,3)$ and computed its divergence in several cases. In the left side of Figure 4 we plot the convergence of the $L^{2}$ norm of this divergence, computed for different degrees and continuities of the discretization space and using the linear $C^{\infty}$ parametrization to describe the physical domain. In the right 

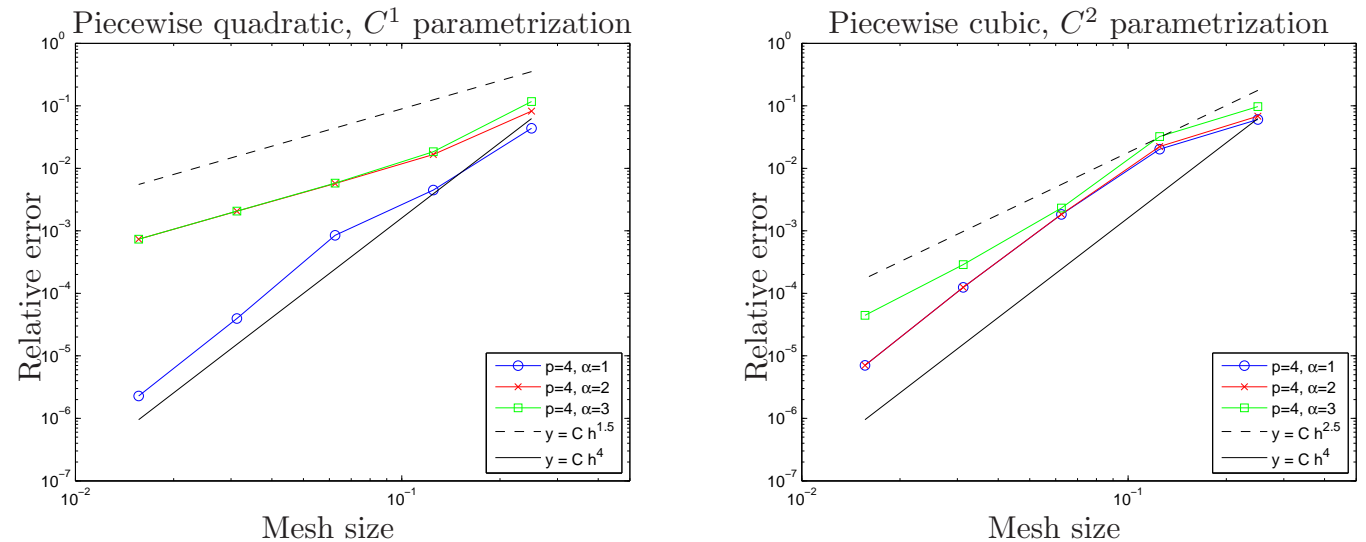

Figure 3: Errors on the $(3,3)$ eigenfunction, in the energy norm on the square. Piecewise quadratic and piecewise cubic parameterizations.

\begin{tabular}{|c|c|c|c|c|c|c|}
\hline Mode & Exact & \multicolumn{5}{|c|}{ Computed } \\
\hline$(1,0)$ & 1.00000 & 1.00060 & 1.00003 & 1.00000 & 1.00000 & 1.00000 \\
\hline$(0,1)$ & 1.00000 & 1.00060 & 1.00003 & 1.00000 & 1.00000 & 1.00000 \\
\hline$(1,1)$ & 2.00000 & 2.00120 & 2.00007 & 2.00000 & 2.00000 & 2.00000 \\
\hline$(2,0)$ & 4.00000 & 4.05285 & 4.00240 & 4.00014 & 4.00001 & 4.00000 \\
\hline$(0,2)$ & 4.00000 & 4.05285 & 4.00240 & 4.00014 & 4.00001 & 4.00000 \\
\hline$(2,1)$ & 5.00000 & 5.05345 & 5.00243 & 5.00014 & 5.00001 & 5.00000 \\
\hline$(1,2)$ & 5.00000 & 5.05345 & 5.00243 & 5.00014 & 5.00001 & 5.00000 \\
\hline$(2,2)$ & 8.00000 & 8.10569 & 8.00480 & 8.00027 & 8.00002 & 8.00000 \\
\hline$(3,0)$ & 9.00000 & 9.79260 & 9.03157 & 9.00162 & 9.00010 & 9.00001 \\
\hline$(0,3)$ & 9.00000 & 9.79260 & 9.03157 & 9.00162 & 9.00010 & 9.00001 \\
\hline$(3,1)$ & 10.00000 & 10.79320 & 10.03160 & 10.00162 & 10.00010 & 10.00001 \\
\hline$(1,3)$ & 10.00000 & 10.79320 & 10.03160 & 10.00162 & 10.00010 & 10.00001 \\
\hline$(3,2)$ & 13.00000 & 13.84545 & 13.03397 & 13.00175 & 13.00010 & 13.00001 \\
\hline$(2,3)$ & 13.00000 & 13.84545 & 13.03397 & 13.00175 & 13.00010 & 13.00001 \\
\hline$(4,0)$ & 16.00000 & 16.21139 & 16.21139 & 16.00960 & 16.00055 & 16.00003 \\
\hline$(0,4)$ & 16.00000 & 16.21139 & 16.21139 & 16.00960 & 16.00055 & 16.00003 \\
\hline$(4,1)$ & 17.00000 & 17.21199 & 17.21142 & 17.00960 & 17.00055 & 17.00003 \\
\hline$(1,4)$ & 17.00000 & 17.21199 & 17.21142 & 17.00960 & 17.00055 & 17.00003 \\
\hline$(3,3)$ & 18.00000 & 19.58520 & 18.06314 & 18.00324 & 18.00019 & 18.00001 \\
\hline$(4,2)$ & 20.00000 & 20.26424 & 20.21379 & 20.00974 & 20.00055 & 20.00003 \\
\hline$(2,4)$ & 20.00000 & 20.26424 & 20.21379 & 20.00974 & 20.00055 & 20.00003 \\
\hline \multicolumn{2}{|c|}{ d.o.f. } & 40 & 144 & 544 & 2112 & 8320 \\
\hline \multicolumn{2}{|c|}{ number of zeros } & 16 & 64 & 256 & 1024 & 4096 \\
\hline
\end{tabular}

Table 1: First non-null eigenvalues computed in the square.

side of Figure 4 we represent the divergence in the particular case of $p=3$ and $\alpha=2$. As can be seen by these results, our method yields an oscillating divergence field, which tends to zero as the mesh is refined.

\subsection{Maxwell's eigenvalues in an L-shaped domain}

For our second test the physical domain is the L-shaped domain $\Omega=(-1,1)^{2} \backslash(-1,0)^{2}$. We describe the geometry by a piecewise quadratic parametrization which is continuously differentiable in the interior of the domain. In order to do so, we introduce the knot vec- 

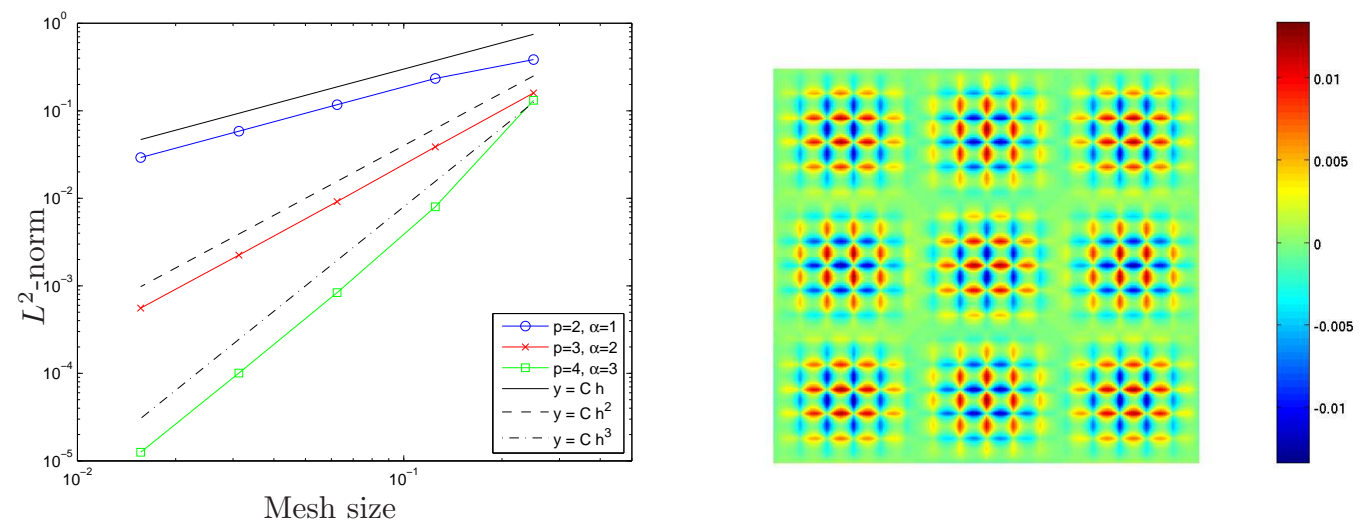

Figure 4: Divergence for the eigenfunction $(m, n)=(3,3)$. Left: convergence of its $L^{2}$-norm. Right: a plot in the case $p=3, \alpha=2$.

tors $\Xi_{1}=\{0,0,0,0.5,1,1,1\}$ and $\Xi_{2}=\{0,0,0,1,1,1\}$, and construct the parametrization by repeating the control points in the corners $(0,0)$ and $(1,1)$ (see Figure 5 ). With this construction, the inverse of the parametrization is unbounded in the two corners $(0,0)$ and $(1,1)$, which is actually a case not covered by the analysis of Section 4 .
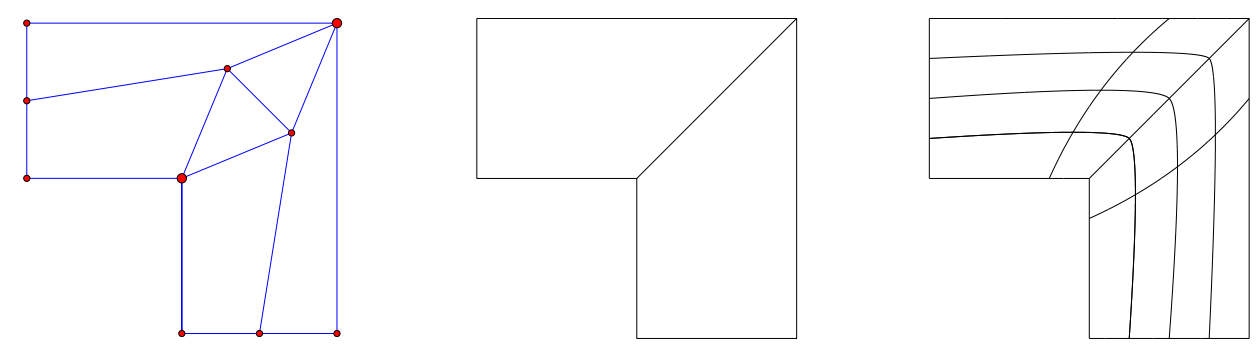

Figure 5: Left: control net for geometry description of the L-shaped domain. Center: mesh generated by the previous control net. Right: coarsest mesh used for computation.

Again, we solve problem (23) in several spaces differing in their degrees and regularity. At the coarsest level of discretization, we consider the spaces $\mathrm{V}_{h ; 0} \equiv \mathrm{V}_{h ; 0}(p, \alpha)$, with $p=$ $1, \ldots, 4$, and internal (global) regularity $\alpha=0$ in the case $p=1$, and $\alpha=1$ in the other three cases. This regularity is achieved by repeating the knot 0.5 in the knot vector $\Xi_{1}$, and it is chosen in order to obtain the optimal convergence, as discussed in Remark 4.1.

The problem is solved in five successively $k$-refined meshes. In Figures 6 and 7 we present the convergence results for the first four non-null eigenvalues. According to [10], the first and second non-null eigenvalues are associated to eigenfunctions which belong to $\mathbf{H}^{2 / 3-\varepsilon}(\Omega)$ and $\mathbf{H}^{4 / 3-\varepsilon}(\Omega)$, respectively, for any $\varepsilon>0$. The convergence rate in energy norm for these functions is expected to be $2 / 3$ and $4 / 3$, respectively, and at least $4 / 3$ and $8 / 3$ for their corresponding eigenvalues. The third and fourth eigenfunctions are analytic, and the convergence of their associated eigenvalues is equal to $p^{2}$ in the four cases. 

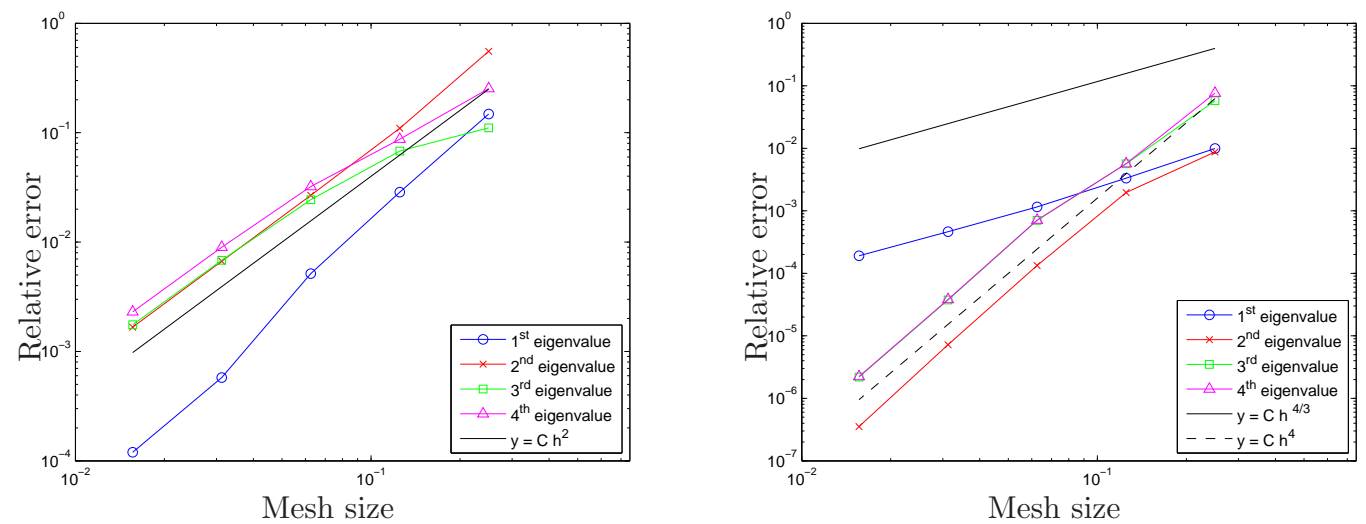

Figure 6: L-shaped domain. Eigenvalues convergence with $p=1, \alpha=0$ (left) and $p=2$, $\alpha=1$ (right).
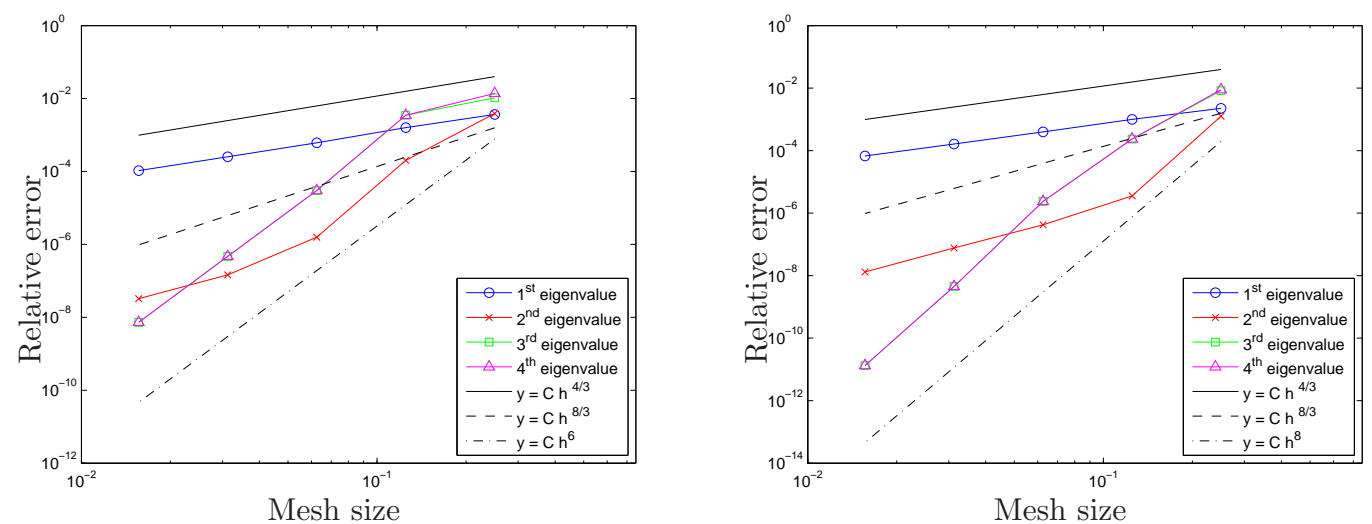

Figure 7: L-shaped domain. Eigenvalues convergence with $p=3, \alpha=1$ (left) and $p=4$, $\alpha=1$ (right)..

In this case it is not possible to know if spurious modes appear in our solution, since the entire spectrum is not known. However, we have compared our results to the ones obtained with other methods (see [6] and [10]) and we can confirm that no spurious solutions appear between our first non-null eigenvalues.

We also present in Table 2 the $L^{2}$-norm of the divergence for the first four eigenfunctions, computed with the choice $p=4$ and $\alpha=1$. It is seen that the norm of divergence for the first eigenfunction increases as we refine the mesh. The same behavior is observed for the divergence of the second eigenfunction, but in this case it is less abrupt. The divergence of the third and fourth eigenfunctions behaves as in the square domain, because these two eigenfunctions are analytic. 


\begin{tabular}{|c||c|c|c|c|c|}
\hline \multicolumn{1}{|c||}{} & \multicolumn{5}{c|}{$L^{2}$-norm of the divergence } \\
\hline Eigf. 1 & $3.09 \mathrm{E}+00$ & $5.60 \mathrm{E}+00$ & $9.87 \mathrm{E}+00$ & $1.76 \mathrm{E}+01$ & $3.14 \mathrm{E}+01$ \\
Eigf. 2 & $4.48 \mathrm{E}-01$ & $3.75 \mathrm{E}-01$ & $3.00 \mathrm{E}-01$ & $4.14 \mathrm{E}-01$ & $5.34 \mathrm{E}-01$ \\
Eigf. 3 & $7.76 \mathrm{E}-02$ & $1.61 \mathrm{E}-01$ & $1.64 \mathrm{E}-03$ & $7.25 \mathrm{E}-05$ & $7.10 \mathrm{E}-06$ \\
Eigf. 4 & $6.05 \mathrm{E}-02$ & $4.59 \mathrm{E}-02$ & $3.78 \mathrm{E}-03$ & $7.40 \mathrm{E}-05$ & $7.04 \mathrm{E}-06$ \\
\hline d.o.f. & 110 & 262 & 758 & 2518 & 9110 \\
\hline
\end{tabular}

Table 2: $L^{2}$-norm of the divergence for the first eigenfunctions in the L-shaped domain.

\subsection{Source problem in an L-shaped domain}

For this third test case we consider the L-shaped domain of the previous section, and denote $\Gamma_{D}:=(-1,0) \times\{0\} \cup\{0\} \times(-1,0)$, and $\Gamma_{N}=\partial \Omega \backslash \bar{\Gamma}_{D}$. We want to solve the problem with mixed boundary conditions given in (24). Denoting by $(r, \theta)$ a system of local polar coordinates, and taking $\mathbf{f}=\operatorname{grad}\left(r^{2 / 3} \sin \frac{2 \theta}{3}\right)$ yields that the unique solution to the problem is given by $\mathbf{u}=\mathbf{f}$. Thus the solution belongs to $\mathbf{H}^{2 / 3-\varepsilon}(\Omega)$, for any $\varepsilon>0$, and the expected convergence rate in the energy norm is then $2 / 3$.

For our computations the geometry is described with the same parametrization given in Section 5.2, and the problem is solved using the same meshes and spaces already described in that section. In Figure 8 we present the convergence rate for the four cases and we see that the convergence rate is always close to $2 / 3$.

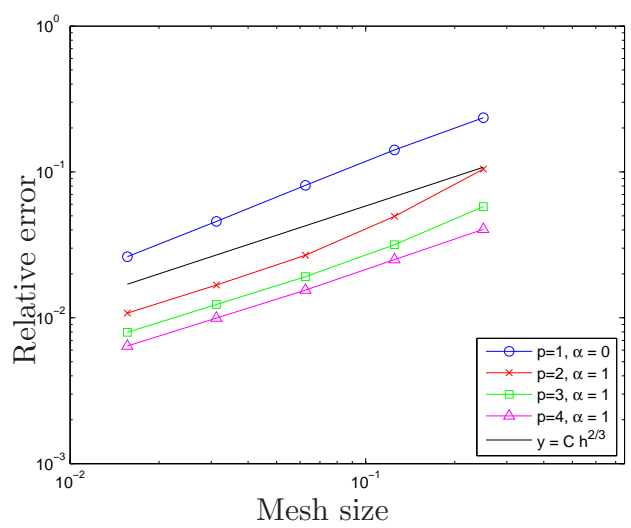

Figure 8: Errors in energy norm for the source problem in the L-shaped domain.

\section{Conclusion}

In this paper, we have proposed a B-spline-based Isogeometric discretization of model electromagnetic problems. To that purpose, we have obtained a De Rham diagram for suitable B-spline spaces, yielding a discretization of $\mathbf{H}(\operatorname{curl} ; \Omega)$ which produces optimal discrete solutions of source and eigenvalue Maxwell's equations. The analysis of the proposed method is not fully developed yet, due to the lack of commuting projectors, which are needed in the so-called De Rham commuting diagram for B-spline spaces. However we have obtained suboptimal convergence results that give a first theoretical assessment of the method. Moreover, we have shown numerical tests that give evidence of an optimal behavior of our approach in 
significant benchmark problems. One interesting feature of our discrete spaces, compared to more classical edge finite elements of Nédélec type, is that they are globally smooth. We can select any order of continuity, with the only constraint that the parametrization of the geometrical domain has to be as smooth as the discrete space used for approximation.

We clearly showed the great potential of the Isogeometric approach in this context, which deserves further theoretical investigation.

\section{Acknowledgments}

The authors were partially supported by the European Research Council through the FP7 Ideas Starting Grant 205004: GeoPDEs - Innovative compatible discretization techniques for Partial Differential Equations. This support is gratefully acknowledged.

\section{References}

[1] D. N. Arnold, R. S. Falk, And R. Winther, Finite element exterior calculus, homological techniques, and applications, Acta Numer., 15 (2006), pp. 1-155.

[2] F. Assous, P. Ciarlet, JR., And J. Segré, Numerical solution to the time-dependent Maxwell equations in two-dimensional singular domains: the singular complement method, J. Comput. Phys., 161 (2000), pp. 218-249.

[3] F. Auricchio, L. B. da Veiga, A. Buffa, C. Lovadina, A. Reali, and G. SanGALLI, A fully "locking-free" isogeometric approach for plane linear elasticity problems: a stream function formulation, Comput. Methods Appl. Mech. Engrg., 197 (2007), pp. 160-172.

[4] Y. Bazilevs, L. Beirão da Veiga, J. A. Cottrell, T. J. R. Hughes, and G. SANGALLI, Isogeometric analysis: approximation, stability and error estimates for $h$-refined meshes, Math. Models Methods Appl. Sci., 16 (2006), pp. 1031-1090.

[5] Y. Bazilevs, V. M. Calo, T. J. R. Hughes, and Y. Zhang, Isogeometric fluidstructure interaction: theory, algorithms, and computations, Comput. Mech., 43 (2008), pp. $3-37$.

[6] D. Boffi, P. Fernandes, L. Gastaldi, And I. Perugia, Computational models of electromagnetic resonators: analysis of edge element approximation, SIAM J. Numer. Anal., 36 (1999), pp. 1264-1290 (electronic).

[7] S. Caorsi, P. Fernandes, and M. Raffetto, On the convergence of Galerkin finite element approximations of electromagnetic eigenproblems, SIAM J. Numer. Anal., 38 (2000), pp. 580-607 (electronic).

[8] M. Costabel, A coercive bilinear form for Maxwell's equations, J. Math. Anal. Appl., 157 (1991), pp. 527-541.

[9] M. Costabel And M. Dauge, Weighted regularization of Maxwell equations in polyhedral domains. A rehabilitation of nodal finite elements, Numer. Math., 93 (2002), pp. 239-277. 
$[10]+$, Computation of resonance frequencies for Maxwell equations in non-smooth domains, in Topics in computational wave propagation, vol. 31 of Lect. Notes Comput. Sci. Eng., Springer, Berlin, 2003, pp. 125-161.

[11] L. B. Da Veiga, A. Buffa, J. Rivas, And G. Sangalli, Some estimates for $h-$ $p-k$-refinement in isogeometric analysis, tech. rep., IMATI-CNR, 2009.

[12] C. DE Boor, A practical guide to splines, vol. 27 of Applied Mathematical Sciences, Springer-Verlag, New York, revised ed., 2001.

[13] J. Evans, Y. Bazilevs, I. Babuska, And T. Hughes, N-widths, sup-infs, and optimality ratios for the $k$-version of the isogeometric finite element method, Comput. Methods Appl. Mech. Engrg., (to appear).

[14] H. Gómez, V. M. Calo, Y. Bazilevs, and T. J. R. Hughes, Isogeometric analysis of the Cahn-Hilliard phase-field model, Comput. Methods Appl. Mech. Engrg., 197 (2008), pp. 4333-4352.

[15] R. D. Graglia And G. Lombardi, Singular higher order complete vector bases for finite methods, IEEE Trans. Antennas and Propagation, 52 (2004), pp. 1672-1685.

[16] T. J. R. Hughes, J. A. Cottrell, and Y. Bazilevs, Isogeometric analysis: CAD, finite elements, NURBS, exact geometry and mesh refinement, Comput. Methods Appl. Mech. Engrg., 194 (2005), pp. 4135-4195.

[17] T. J. R. Hughes, A. Reali, And G. SAngalli, Duality and unified analysis of discrete approximations in structural dynamics and wave propagation: comparison of p-method finite elements with $k$-method NURBS, Comput. Methods Appl. Mech. Engrg., 197 (2008), pp. 4104-4124.

[18] P. Monk, Finite Element Methods for Maxwell's Equations, Oxford University Press, Oxford, 2003.

[19] J.-C. NÉDÉLEC, Mixed finite elements in $R^{3}$, Numer. Math., 35 (1980), pp. 315-341.

[20] L. Piegl And W. Tiller, The Nurbs Book, Springer-Verlag, New York, 1997.

[21] L. L. Schumaker, Spline functions: basic theory, Cambridge Mathematical Library, Cambridge University Press, Cambridge, third ed., 2007. 